\title{
ANALYTIC SOLUTIONS FOR SINGULAR INTEGRAL EQUATIONS AND NON-HOMOGENEOUS FRACTIONAL PDE
}

\author{
ARMAN AGHILI
}

\begin{abstract}
In the last three decades, transform methods have been used for solving fractional differential equations, singular integral equations. In this article, the author considered a new class of the inverse Laplace transforms of exponential types. We also evaluated certain types of integrals and solved partial fractional equations of Cauchy type.The result reveals that the transform method is very convenient and effective.
\end{abstract}

\section{INTRODUCTION AND PRELIMINARIES}

In this paper, the author implemented transform method for solving singular integral equations and partial fractional differential equation which arises in applications. Several methods have been introduced to solve fractional differential equations, the popular Laplace transform method $[1-3,6]$, the Fourier transform method [14], the iteration method [13] and operational method [7, 9, 10]. However, most of these methods are suitable for special types of fractional differential equations, mainly the linear with constant coefficients. More detailed information about some of these results can be found in a survey paper by Kilbas, Srivastava and Trujillo [8]. Atanacković and Stanković [4,5] and Stanković [17] used the Laplace transform in a certain space of distributions to solve a system of partial differential equations with fractional derivatives, and indicated that such a system may serve as a certain model for a visco-elastic rod. Oldham and Spanier [11,12], by reducing a boundary value problem involving Fick's second law in electro analytic chemistry obtained a formulation based on the partial Riemann-Liouville fractional derivative of order $1 / 2$. Wyss [19] and Schneider [16] considered the time fractional diffusion and wave equations and obtained the solution in terms of Fox functions. In the last section of this paper, we consider four-term time fractional non homogeneous sub ballistic partial differential equation (time fractional in the Caputo sense).

$M S C$ (2010): primary 26A33, 34A08, 34K37, 35R11.

Keywords: Laplace transforms, Caputo fractional derivative, nonhomogeneous time fractional partial differential equation, singular integral equations. 


\subsection{Definitions and notations}

Definition 1.1. The left Caputo fractional derivative of order $\alpha(0<\alpha<1)$ of $\phi(t)$ is defined as follows [13]

$$
D_{a}^{c, \alpha} \phi(x)=\frac{1}{\Gamma(1-\alpha)} \int_{a}^{t} \frac{1}{(t-\xi)^{\alpha}} \phi^{\prime}(\xi) d \xi .
$$

Definition 1.2. The Laplace transform of a function $f(t)$ is defined as follows

$$
\mathcal{L}\{f(t)\}=\int_{0}^{\infty} e^{-s t} f(t) d t=F(s) .
$$

If $\mathcal{L}\{f(t)\}=F(s)$, then $\mathcal{L}^{-1}\{F(s)\}$ is given by

$$
f(t)=\frac{1}{2 \pi i} \int_{c-i \infty}^{c+i \infty} e^{s t} F(s) d s
$$

where $F(s)$ is analytic in the region $\operatorname{Re}(s)>c[15]$.

The most important use of the Caputo fractional derivative is treated in initial value problems where the initial conditions are expressed in terms of integer order derivatives. In this respect, it is interesting to know the Laplace transform of this kind of derivative. Using the Laplace transforms of Caputo fractional derivatives of a non-integer order $0<\alpha \leq 1$, we get $[8,14]$

$$
\mathcal{L}\left\{D_{a}^{c, \alpha} f(t)\right\}=s F(s)-f(0+), \quad 0<\alpha<1,
$$

and generally [14]

$$
\mathcal{L}\left\{D_{a}^{c, \alpha} f(t)\right\}=s^{\alpha-1} F(s)-\sum_{k=0}^{k=m-1} s^{\alpha-1-k} f^{k}(0+), \quad m-1<\alpha<m .
$$

The Laplace transform provides a useful technique for the solution of such fractional singular integro-differential equations.

Definition 1.3. The two-parameter function of the Mittag-Leffler type is defined by the following series expansion [8]

$$
E_{\alpha, \beta}(z)=\sum_{n=0}^{\infty} \frac{z^{n}}{\Gamma(\alpha n+\beta)} .
$$

The simplest Wright function is given by the series

$$
W(\alpha, \beta ; z)=\sum_{n=0}^{\infty} \frac{z^{n}}{n ! \Gamma(\alpha n+\beta)},
$$

when $\alpha, \beta, z \in \mathbb{C}$. We have the following relationship [14]

$$
\mathcal{L}\left\{t^{\beta-1} E_{\alpha, \beta}\left( \pm a t^{\alpha}\right)\right\}=\frac{s^{\alpha-\beta}}{s^{\alpha} \mp a} \quad\left(\operatorname{Re}(s)>|a|^{\frac{1}{\alpha}}\right) .
$$

Generalizations of the Mittag-Leffler function (1.1) to two variables were further extended by H. M. Srivastava. 
Definition 1.4. The error function is defined by the integral

$$
\operatorname{Erf}(t)=\frac{2}{\sqrt{\pi}} \int_{0}^{t} e^{-u^{2}} d u
$$

This function is encountered in the theory of errors, the theory of heat conduction, and various branches of engineering and mathematical physics.

Definition 1.5. The complementary error function is defined by the integral

$$
\operatorname{Erfc}(t)=\frac{2}{\sqrt{\pi}} \int_{t}^{\infty} e^{-u^{2}} d u=1-\operatorname{Erf}(t)
$$

Lemma 1.6. (Efros's theorem) Assume that $\mathcal{L}\{f(t)\}=F(s)$ and $\mathcal{L}\{u(t, \tau)\}=$ $U(s) \exp (-\tau q(s))$ where $U(s), q(s)$ are analytic. Then, we have

$$
\mathcal{L}\left\{\int_{0}^{\infty} f(\tau) u(t, \tau) d \tau\right\}=U(s) F(q(s))
$$

Proof. See $[3,7]$.

Corollary 1.7. Let $L\{f(t)\}=F(s)$. Then, the following identities hold true.

(1) $\mathcal{L}^{-1}\left\{F\left(s^{\alpha}\right)\right\}=\frac{1}{\pi} \int_{0}^{\infty} f(u) \int_{0}^{\infty} e^{-t r-u r^{\alpha} \cos \alpha \pi} \sin \left(u r^{\alpha} \sin \alpha \pi\right) d r d u$,

(2) $\mathcal{L}^{-1}\left\{\frac{F(\sqrt{s})}{\sqrt{s}}\right\}=\frac{1}{\sqrt{\pi t}} \int_{0}^{\infty} e^{-\frac{u^{2}}{4 t}} f(u) d u$,

(3) $\mathcal{L}^{-1}\{F(\sqrt{s})\}=\frac{1}{2 t \sqrt{\pi t}} \int_{0}^{\infty} u e^{-\frac{u^{2}}{4 t}} f(u) d u$,

(4) $\mathcal{L}^{-1}\left\{\frac{1}{\sqrt{s}(\sqrt{s}+a)}\right\}=e^{a^{2} t} \operatorname{Erfc}(a \sqrt{t})$.

(5) $\mathcal{L}^{-1}\left\{\frac{e^{-\lambda s^{\alpha}}}{s}\right\}=\frac{1}{\pi} \int_{0}^{\infty} \frac{e^{-t r-\lambda r^{\alpha} \cos \pi \alpha}}{r} \sin \left(\lambda r^{\alpha} \sin (\pi \alpha)\right) d r$.

Proof. See $[1,3]$.

Example 1.8. If we set $U(s)=s^{-\alpha}$ and $q(s)=s^{\alpha}$, then we get

This leads to

$$
\mathcal{L}\{u(t, \tau)\}=U(s) \exp (-\tau q(s))=\frac{\exp \left(-\tau s^{\alpha}\right)}{s^{\alpha}} .
$$

Thus, we obtain

$$
u(t, \tau)=t^{\alpha-1} W\left(-\alpha, \alpha: \tau t^{-\alpha}\right) .
$$

$$
\mathcal{L}\left\{\int_{0}^{\infty} f(\tau) u(t, \tau) d \tau\right\}=\mathcal{L}\left\{t^{\alpha-1} \int_{0}^{\infty} f(\tau) W\left(-\alpha, \alpha: \tau t^{-\alpha}\right) d \tau\right\}=\frac{F\left(s^{\alpha}\right)}{s^{\alpha}}
$$

Lemma 1.9. We have the following integral relation

$$
\int_{0}^{1} \operatorname{Erf}(\sin \phi) \operatorname{Erf}(\cos \phi) \sin 2 \phi d \phi=1 .
$$

Proof. Let us take $f(t)=\operatorname{Erf}(\sqrt{t})$, then we have $\mathcal{L}\{f(t)\}=F(s)=\frac{1}{s \sqrt{s+1}}$, define

$$
G(s)=F(s) F(s)=\frac{1}{s^{2}(s+1)},
$$

at this point, we invert $G(s)$ in two different ways

$$
\mathcal{L}^{-1}\{G(s)\}=\mathcal{L}^{-1}\left\{\frac{1}{s^{2}(s+1)}\right\}=t e^{-t}-e^{-t}+1,
$$


and, by the convolution of the Laplace transform, one gets the following sequence of relations

$$
\begin{aligned}
\mathcal{L}^{-1}\{G(s)\}=\mathcal{L}^{-1}\{F(s) F(s)\} & =f(t) * f(t) \\
= & \operatorname{Erf}(\sqrt{t}) * \operatorname{Erf}(\sqrt{t})=\int_{0}^{t} \operatorname{Erf}(\sqrt{\xi}) \operatorname{Erf}(\sqrt{t-\xi}) d \xi .
\end{aligned}
$$

Finally, we obtain

$$
\mathcal{L}^{-1}\{F(s) F(s)\}=\int_{0}^{t} \operatorname{Erf}(\sqrt{\xi}) \operatorname{Erf}(\sqrt{t-\xi}) d \xi=t e^{-t}-e^{-t}+1,
$$

Taking $t=1$ and making the change of variable $\xi=\sin ^{2} \phi$, after simplifying, we arrive at

$$
\int_{0}^{1} \operatorname{Erf}(\sin \phi) \operatorname{Erf}(\cos \phi) \sin 2 \phi d \phi=1
$$

\subsection{Solution to singular integral equation with trigonometric kernel}

The Laplace transforms can be used to solve certain types of singular integral equations. Singular integral equations arise in many problems of mathematical physics. The mathematical formulation of physical phenomena often involves Cauchy type, or more severe, singular integral equations [15]. Applications in many important fields, such as fracture mechanics, elastic contact problems, the theory of porous filtering contain integral and integro-differential equations with singular kernel.

Lemma 1.10. Let us assume that $\mathcal{L} f(t)=F(s)$, then the following relations hold true

$$
\begin{aligned}
& \text { 1. } \mathcal{L}\left\{\int_{0}^{\infty} \frac{\sin (2 \sqrt{t \tau})}{\sqrt{\tau}} f(\tau) d \tau\right\}=\sqrt{\frac{\pi}{s^{3}}} F\left(\frac{1}{s}\right), \\
& \text { 2. } \mathcal{L}\left\{\int_{0}^{\infty} \frac{\cos (2 \sqrt{t \tau})}{\sqrt{t}} f(\tau) d \tau\right\}=\sqrt{\frac{\pi}{s}} F\left(\frac{1}{s}\right) .
\end{aligned}
$$

Proof. See [3].

Lemma 1.11. Let us consider the following singular integral equation of the form

$$
f(x)=g(x)+\lambda \int_{0}^{\infty} \frac{\sin (2 \sqrt{x \tau})}{\sqrt{\tau}} f(\tau) d \tau, \quad \lambda \neq 0, \pm 1,
$$

where $g(x)$ is a function with the Laplace transform $G(s)$. Then, the above singular integral equation has the following formal solution

$$
f(x)=\frac{1}{2 i \pi} \int_{c-i \infty}^{c+i \infty} \frac{G(s)+\frac{\lambda}{s \sqrt{s}} G\left(\frac{1}{s}\right)}{1-\lambda^{2}} e^{x s} d s .
$$

Proof. Let $F(s), G(s)$ be the Laplace transforms of $f(x)$ and $g(x)$, respectively. Then, taking the Laplace transform of the integral equation term wise and in view of part one of the above Lemma 1.10, we get the following relation,

$$
F(s)=G(s)+\lambda \sqrt{\frac{\pi}{s^{3}}} F\left(\frac{1}{s}\right) .
$$


Now, in the above relation we replace $s$ by $\frac{1}{s}$ to obtain

$$
F\left(\frac{1}{s}\right)=G\left(\frac{1}{s}\right)+\lambda s \sqrt{\pi s} F(s) .
$$

A combination of (1.3) and (1.2) and calculation of $F(s)$ leads to the following,

$$
F(s)=\frac{G(s)+\frac{\lambda}{s \sqrt{s}} G\left(\frac{1}{s}\right)}{1-\lambda^{2}},
$$

upon using complex inversion formula, relation (1.4) reads

$$
f(x)=\frac{1}{2 i \pi} \int_{c-i \infty}^{c+i \infty} \frac{G(s)+\frac{\lambda}{s \sqrt{s}} G\left(\frac{1}{s}\right)}{1-\lambda^{2}} e^{x s} d s .
$$

Example 1.12. Let us solve the following singular integral equation.

$$
f(x)=e^{-a x}+\lambda \int_{0}^{\infty} \frac{\sin (2 \sqrt{x \tau})}{\sqrt{\tau}} f(\tau) d \tau, \quad \lambda \neq 0, \pm 1 .
$$

Solution. Taking the Laplace transform of the above integral equation term wise, leads to the following equation

$$
F(s)=\frac{\frac{1}{s+a}+\frac{\lambda}{s \sqrt{s}} \frac{s}{a s+1}}{1-\lambda^{2}}
$$

therefore, the final solution is

$$
f(x)=\frac{1}{2 i \pi} \int_{c-i \infty}^{c+i \infty} \frac{\frac{1}{s+a}+\frac{\lambda}{\sqrt{s}} \frac{1}{a s+1}}{1-\lambda^{2}} e^{x s} d s,
$$

or,

$$
f(x)=\frac{e^{-a x}}{1-\lambda^{2}}+\frac{\lambda}{a\left(1-\lambda^{2}\right)} \frac{1}{\sqrt{\pi x}} * e^{-\frac{x}{a}},
$$

equivalently

$$
f(x)=\frac{e^{-a x}}{1-\lambda^{2}}+\frac{\lambda}{a\left(1-\lambda^{2}\right)} \int_{0}^{x} \frac{e^{-\frac{\xi}{a}}}{\sqrt{\pi(x-\xi)}} d \xi .
$$

Lemma 1.13. Let us consider the following fractional singular integral equation $D_{x}^{c, \alpha} f(x)=g(x)+\lambda \int_{0}^{\infty} \frac{\sin (2 \sqrt{x \tau})}{\sqrt{\tau}} f(\tau) d \tau, \quad \lambda \neq 0, \pm 1, \quad 0<\alpha \leq 1, \quad f(0)=0$.

The above integral equation has the formal solution as

$$
f(x)=\frac{1}{2 i \pi} \int_{c-i \infty}^{c+i \infty} \frac{s^{\alpha-1} G(s)+\frac{\lambda}{s \sqrt{s}} G\left(\frac{1}{s}\right)}{1-\lambda^{2}} e^{x s} d s,
$$

equivalently

$$
f(x)=\frac{1}{\left(1-\lambda^{2}\right) \Gamma(\alpha)} x^{1-\alpha} * g(x)+\lambda \int_{0}^{\infty} \frac{\sin (2 \sqrt{x \tau})}{\sqrt{\tau}} g(\tau) d \tau .
$$


Proof. Let $F(s), G(s)$ be the Laplace transforms of $f(x), g(x)$, respectively, then we get the following relation,

$$
s^{\alpha} F(s)=G(s)+\lambda \sqrt{\frac{\pi}{s^{3}}} F\left(\frac{1}{s}\right) .
$$

Now, in the above relation, we replace $s$ by $\frac{1}{s}$ to obtain

$$
s^{-\alpha} F\left(\frac{1}{s}\right)=G\left(\frac{1}{s}\right)+\lambda s \sqrt{\pi s} F(s) .
$$

A combination of (1.5) and (1.6) and calculation of $F(s)$ leads to the following,

$$
F(s)=\frac{s^{-\alpha} G(s)+\frac{\lambda}{s \sqrt{s}} G\left(\frac{1}{s}\right)}{1-\lambda^{2}}
$$

upon using complex inversion formula, relation (1.7) reads,

$$
f(x)=\frac{1}{2 \pi i} \int_{c-i \infty}^{c+\infty} \frac{s^{-\alpha} G(s)+\frac{\lambda}{s \sqrt{s}} G\left(\frac{1}{s}\right)}{1-\lambda^{2}} e^{x s} d s .
$$

Example 1.14. Let us solve the following fractional singular integro-differential equation,

$$
D_{x}^{c, 0.5} f(x)=\sin \beta x+\lambda \int_{0}^{\infty} \frac{\sin (2 \sqrt{x \tau})}{\sqrt{\tau}} f(\tau) d \tau, \quad \lambda \neq 0, \pm 1, \quad f(0)=0 .
$$

Solution. Using relation (1.8) leads to the following formal solution

$$
f(x)=\frac{1}{2 \pi i} \int_{c-i \infty}^{c+\infty} \frac{s^{-0.5}\left(\frac{\beta}{s^{2}+\beta^{2}}\right)+\frac{\lambda}{s \sqrt{s}}\left(\frac{\beta s^{2}}{\beta^{2} s^{2}+1}\right)}{1-\lambda^{2}} e^{x s} d s,
$$

finally, we obtain the solution as below

$$
f(x)=\frac{1}{1-\lambda^{2}} \int_{0}^{x} \frac{1}{\sqrt{\pi(x-\xi)}}\left(\beta \sin \beta \xi+\frac{\lambda}{\beta} \cos \left(\frac{\xi}{\beta}\right)\right) d \xi .
$$

Theorem 1.15. Let us consider the system of time fractional Fredholm singular integro-differential equations, where $g_{1}(t), g_{2}(t)$, are Laplace transformable functions with the Laplace transforms $G_{1}(s), G_{2}(s)$, respectively and

$$
\begin{aligned}
& D_{x}^{c, \alpha} \phi(x)=g_{1}(x)+\lambda_{1} \int_{0}^{\infty} \frac{\cos (2 \sqrt{x \tau})}{\sqrt{x}} \psi(\tau) d \tau, \quad \lambda_{1} \neq 0, \pm 1,0<\alpha \leq 1, \phi(0)=0 . \\
& D_{x}^{c, \beta} \psi(x)=g_{2}(x)+\lambda_{2} \int_{0}^{\infty} \frac{\sin (2 \sqrt{x \tau})}{\sqrt{\tau}} \phi(\tau) d \tau, \quad \lambda_{2} \neq 0, \pm 1,0<\beta \leq 1, \quad \psi(0)=0 .
\end{aligned}
$$

Then, the above system has the following formal solutions

$$
\begin{aligned}
& \phi(x)=-\sum_{k=0}^{\infty} \frac{\left(\pi \lambda_{1} \lambda_{2}\right)^{-(k+1)} g_{1}(x) * x^{k(\beta-\alpha+2)+\beta+1)}}{\Gamma(k(\beta-\alpha+2)+\beta+2)} \\
&-\sqrt{\pi} \lambda_{1} \sum_{k=0}^{\infty} \frac{\left(\pi \lambda_{1} \lambda_{2}\right)^{-(k+1)} R(x)}{\Gamma(k(\beta-\alpha+2)+1)},
\end{aligned}
$$




$$
\begin{aligned}
& \psi(x)=\sum_{k=0}^{\infty} \frac{\left(\pi \lambda_{1} \lambda_{2}\right)^{k} g_{2}(x) * x^{k(\beta-\alpha+2.5)+\beta-1}}{\Gamma(k(\beta-\alpha+2.5)+\beta)} \\
& \quad+\sqrt{\pi} \lambda_{2} \sum_{k=0}^{\infty} \frac{\left(\pi \lambda_{1} \lambda_{2}\right)^{k} Q(x)}{\Gamma(k(\beta-\alpha+2)+\beta-\alpha+1.5)},
\end{aligned}
$$

where $R(x)$ and $Q(x)$ are as follows

$$
\begin{gathered}
R(x)=x^{k(\beta-\alpha+2))} * \int_{0}^{\infty} \frac{\sin (2 \sqrt{x \xi})}{\xi} g_{2}(\xi) d \xi \\
Q(x)=x^{k(\beta-\alpha+2)+\beta-\alpha+0.5)} * \int_{0}^{\infty} \frac{\cos (2 \sqrt{x \xi})}{\xi} g_{1}(\xi) d \xi
\end{gathered}
$$

In the above relation, $*$ stands for the convolution product.

Proof. Taking the Laplace transform of the above system term-wise and using Lemma 1.10 and initial conditions leads to the following relations

$$
\begin{gathered}
s^{\alpha} \Phi(s)=G_{1}(s)+\lambda \sqrt{\frac{\pi}{s}} \Psi\left(\frac{1}{s}\right), \\
s^{\beta} \Psi(s)=G_{2}(s)+\lambda \sqrt{\frac{\pi}{s^{3}}} \Phi\left(\frac{1}{s}\right) .
\end{gathered}
$$

Now, in the above relations, we replace $\mathrm{s}$ by $\frac{1}{s}$, to obtain

$$
\begin{aligned}
& s^{-\alpha} \Phi\left(\frac{1}{s}\right)=G_{1}\left(\frac{1}{s}\right)+\lambda_{1} \sqrt{\pi s} \Psi(s), \\
& s^{-\beta} \Psi\left(\frac{1}{s}\right)=G_{2}\left(\frac{1}{s}\right)+\lambda_{2} s \sqrt{\pi s} \Phi(s) .
\end{aligned}
$$

At this point, by inserting relations (1.11), (1.12) in (1.10)) and (1.9), respectively, we get the following equations

$$
\begin{gathered}
s^{\alpha} \Phi(s)=G_{1}(s)+\lambda_{1} \sqrt{\frac{\pi}{s}} s^{\beta}\left(G_{2}\left(\frac{1}{s}\right)+\lambda_{2} s \sqrt{\pi s} \Phi(s)\right), \\
s^{\beta} \Psi(s)=G_{2}(s)+\lambda \sqrt{\frac{\pi}{s^{3}}} s^{\alpha}\left(G_{1}\left(\frac{1}{s}\right)+\lambda_{1} \sqrt{\pi s} \Psi(s)\right),
\end{gathered}
$$

finally, we arrive at the following

$$
\begin{aligned}
& \Phi(s)=\frac{G_{1}(s)+\lambda_{1} \sqrt{\pi} s^{\beta-0.5} G_{2}\left(\frac{1}{s}\right)}{s^{\alpha}-\pi \lambda_{1} \lambda_{2} s^{\beta+2}}, \\
& \Psi(s)=\frac{G_{2}(s)+\lambda_{2} \sqrt{\pi} s^{\alpha-2.5} G_{1}\left(\frac{1}{s}\right)}{s^{\beta}-\pi \lambda_{1} \lambda_{2} s^{\alpha-2.5}} .
\end{aligned}
$$

Further, simplifying yields

$$
\begin{aligned}
& \Phi(s)=\frac{1}{s^{\alpha}-\pi \lambda_{1} \lambda_{2} s^{\beta+2}} G_{1}(s)+\frac{\lambda_{1} \sqrt{\pi} s^{\beta+1}}{s^{\alpha}-\pi \lambda_{1} \lambda_{2} s^{\beta+2}}\left(\frac{1}{s \sqrt{s}} G_{2}\left(\frac{1}{s}\right)\right), \\
& \Psi(s)=\frac{1}{s^{\beta}-\pi \lambda_{1} \lambda_{2} s^{\alpha-2.5}} G_{2}(s)+\frac{\lambda_{2} \sqrt{\pi} s^{\alpha-1.5}}{s^{\beta}-\pi \lambda_{1} \lambda_{2} s^{\alpha-2.5}}\left(\frac{1}{s} G_{1}\left(\frac{1}{s}\right)\right) .
\end{aligned}
$$


Let us make series expansions of all fractions in the above relations to obtain

$$
\begin{gathered}
\Phi(s)=-G_{1}(s) \sum_{k=0}^{\infty} \frac{\left(\pi \lambda_{1} \lambda_{2}\right)^{-(k+1)}}{s^{k(\beta-\alpha+2)+\beta+2}}-\sqrt{\pi} \lambda_{1}\left(\frac{1}{s \sqrt{s}} G_{2}\left(\frac{1}{s}\right)\right) \sum_{k=0}^{\infty} \frac{\left(\pi \lambda_{1} \lambda_{2}\right)^{-(k+1)}}{s^{k(\beta-\alpha+2)+1}}, \\
\Psi(s)=G_{2}(s) \sum_{k=0}^{\infty} \frac{\left(\pi \lambda_{1} \lambda_{2}\right)^{k}}{s^{k(\beta-\alpha+2.5)+\beta}}+\sqrt{\pi} \lambda_{2}\left(\frac{1}{s} G_{1}\left(\frac{1}{s}\right)\right) \sum_{k=0}^{\infty} \frac{\left(\pi \lambda_{1} \lambda_{2}\right)^{k}}{s^{k(\beta-\alpha+2)+\beta-\alpha+1.5}}
\end{gathered}
$$

Taking the inverse Laplace transforms term wise leads to the following formal solutions

$$
\begin{aligned}
\phi(x)= & -\sum_{k=0}^{\infty} \frac{\left(\pi \lambda_{1} \lambda_{2}\right)^{-(k+1)} g_{1}(x) * x^{k(\beta-\alpha+2)+\beta+1)}}{\Gamma(k(\beta-\alpha+2)+\beta+2)} \\
& -\sqrt{\pi} \lambda_{1} \sum_{k=0}^{\infty} \frac{\left(\pi \lambda_{1} \lambda_{2}\right)^{-(k+1)} R(x)}{\Gamma(k(\beta-\alpha+2)+1)}, \\
\psi(x)= & \sum_{k=0}^{\infty} \frac{\left(\pi \lambda_{1} \lambda_{2}\right)^{k} g_{2}(x) * x^{k(\beta-\alpha+2.5)+\beta-1}}{\Gamma(k(\beta-\alpha+2.5)+\beta)} \\
& +\sqrt{\pi} \lambda_{2} \sum_{k=0}^{\infty} \frac{\left(\pi \lambda_{1} \lambda_{2}\right)^{k} Q(x)}{\Gamma(k(\beta-\alpha+2)+\beta-\alpha+1.5)},
\end{aligned}
$$

where $R(x)$ and $Q(x)$ are as follows

$$
\begin{gathered}
R(x)=x^{k(\beta-\alpha+2))} * \int_{0}^{\infty} \frac{\sin (2 \sqrt{x \xi})}{\xi} g_{2}(\xi) d \xi \\
Q(x)=x^{k(\beta-\alpha+2)+\beta-\alpha+0.5)} * \int_{0}^{\infty} \frac{\cos (2 \sqrt{x \xi})}{\xi} g_{1}(\xi) d \xi
\end{gathered}
$$

\subsection{Inverse Laplace transforms of exponential functions involving nested square roots}

Let us pass to a new class of inverse Laplace transforms of exponential functions involving nested square roots. The inverse Laplace transforms involving nested square roots arise in many areas of applied mathematics, usually as a result of linear evolution partial differential equations in fourth order in the spatial variables. Examples of such problems abound in fluid mechanics.

Lemma 1.16. The following relation holds true

$$
\mathcal{L}^{-1}\left\{\exp \left(-\lambda \sqrt{2 a s+2 \sqrt{a^{2} s^{2}-k^{2}}}\right\}=\frac{a \lambda^{2} e^{-\frac{k t}{a}}}{4 \pi} \int_{0}^{t} \frac{e^{\frac{2 k \xi}{a}-\frac{a t \lambda^{2}}{4 \xi(t-\xi)}}}{\sqrt{(t-\xi)^{3}}} d \xi .\right.
$$

Proof. Let us assume that $F(s)=\exp \left(-\lambda \sqrt{2 a s+2 \sqrt{a^{2} s^{2}-k^{2}}}\right)$, then $F(s)$ can be rewritten as follows,

$$
F(s)=\exp \left(-\lambda \sqrt{2 a s+2 \sqrt{a^{2} s^{2}-k^{2}}}\right)=\exp (-\lambda \sqrt{a s+k}) \exp (-\lambda \sqrt{a s-k}) .
$$


On the other hand, from the Laplace transforms table, we have the following relations

$$
\mathcal{L}^{-1}\left\{\exp (-\lambda \sqrt{a s \pm k}\}=\frac{\lambda \sqrt{a}}{2 t \sqrt{\pi t}} \exp \left(\mp \frac{k t}{a}-\frac{a \lambda^{2}}{4 t}\right) .\right.
$$

Finally, we have

$$
\begin{aligned}
\mathcal{L}^{-1}\left\{\exp \left(-\lambda \sqrt{2 a s+2} \sqrt{a^{2} s^{2}-k^{2}}\right)\right\} \\
=\mathcal{L}^{-1}\{\exp (-\lambda \sqrt{a s+k})\} * \mathcal{L}^{-1}\{\exp (-\lambda \sqrt{a s-k})\},
\end{aligned}
$$

hence,

$$
\begin{aligned}
\mathcal{L}^{-1}\{F(s)\}=\int_{0}^{t} & \frac{\lambda \sqrt{a}}{2(t-\xi) \sqrt{\pi(t-\xi)}} \\
& \quad \exp \left(-\frac{k(t-\xi)}{a}-\frac{a \lambda^{2}}{4(t-\xi)}\right) \frac{\lambda \sqrt{a}}{2 \xi \sqrt{\pi \xi}} \exp \left(\frac{k \xi}{a}-\frac{a \lambda^{2}}{4 \xi}\right) d \xi .
\end{aligned}
$$

Further, simplifying leads to the following result

$$
\mathcal{L}^{-1}\{F(s)\}=\frac{a \lambda^{2} e^{-\frac{k t}{a}}}{4 \pi} \int_{0}^{t} \frac{e^{\frac{2 k \xi}{a}-\frac{a t \lambda^{2}}{4 \xi(t-\xi)}}}{\sqrt{(t-\xi)^{3}}} d \xi
$$

Lemma 1.17. By using complex inversion formula for the Laplace transforms, we get

$$
\begin{aligned}
& \text { 1. } \mathcal{L}^{-1}\left\{\frac{e^{-\frac{k^{2}}{4 s}}}{\sqrt{s}}\right\}=\frac{\cos k \sqrt{t}}{\sqrt{\pi t}} \\
& \text { 2. } \mathcal{L}^{-1}\left\{\frac{e^{-\frac{k^{2}}{4(\sqrt{\lambda+\sqrt{s}})}}}{\sqrt{\lambda+\sqrt{s}}}\right\}=\frac{1}{2 t \sqrt{\pi t}} \int_{0}^{\infty} w e^{-\frac{w^{2}}{4 t}} \\
& \left(\frac{e^{-\lambda w}}{2 w \sqrt{\pi w}} \int_{0}^{\infty} u e^{\frac{-u^{2}}{4 w}} J_{0}(k \sqrt{u}) d u\right) d w .
\end{aligned}
$$

Proof. 1. Direct application of complex inversion formula leads to the following relation,

$$
f(t)=\mathcal{L}^{-1}\left\{\frac{e^{-\frac{k^{2}}{4 s}}}{\sqrt{s}}\right\}=\frac{1}{2 \pi i} \int_{c-i \infty}^{c+i \infty}\left(\frac{e^{-\frac{k^{2}}{4 s}}}{\sqrt{s}}\right) e^{t s} d s .
$$

In order to calculate the above complex integral, we may use the following integral representation for the integrand,

$$
\frac{e^{-\frac{k^{2}}{4 s}}}{\sqrt{s}}=\frac{2}{\sqrt{\pi}} \int_{0}^{\infty} e^{-\frac{a u^{2}}{k^{2}}} \cos u d u
$$

At this point, if we set (1.14) in (1.13), we get the following

$$
f(t)=\mathcal{L}^{-1}\left\{\frac{e^{-\frac{k^{2}}{4 s}}}{\sqrt{s}}\right\}=\frac{1}{2 \pi i} \int_{c-i \infty}^{c+i \infty}\left(\frac{2}{\sqrt{\pi}} \int_{0}^{\infty} e^{-\frac{s u^{2}}{k^{2}}} \cos u d u\right) e^{t s} d s .
$$


By changing the order of integration which is permissible, we obtain

$$
\begin{aligned}
f(t)=\int_{0}^{\infty} \cos u\left(\frac{1}{2 \pi i} \int_{c-i \infty}^{c+i \infty} e^{t s} e^{-\frac{s u^{2}}{k^{2}}} d s\right) d u \\
\quad=\int_{0}^{\infty} \cos u\left(\frac{1}{2 \pi i} \int_{c-i \infty}^{c+i \infty} e^{\left(t-\frac{u^{2}}{k^{2}}\right) s} d s\right) d u
\end{aligned}
$$

But the value of the inner complex integral is $\delta\left(t-\frac{u^{2}}{k^{2}}\right)$, therefore, we get

$$
f(t)=\int_{0}^{\infty} \delta\left(t-\frac{u^{2}}{k^{2}}\right) \cos u d u .
$$

If we introduce the new change of variable $t-\frac{u^{2}}{k^{2}}=\eta$ in the above integral and use the well-known elementary properties of Dirac delta function, we get

$$
f(t)=\frac{1}{\sqrt{\pi}} \int_{-\infty}^{t} \delta(\eta) \frac{\cos (\sqrt{t-\eta})}{\sqrt{t-\eta}} d \eta=\frac{\cos k \sqrt{t}}{\sqrt{\pi t}} .
$$

2. Let us define

$$
G(s)=\frac{e^{-\frac{k^{2}}{4 \sqrt{s+\lambda}}}}{\sqrt{s+\lambda}}
$$

we have

$$
\mathcal{L}^{-1}\{G(s)\}=\frac{e^{-\lambda t}}{2 t \sqrt{\pi t}} \int_{0}^{\infty} u e^{\frac{-u^{2}}{4 t}} J_{0}(k \sqrt{u}) d u .
$$

By using Efros's theorem or Corollary 1.7, we get the following

$$
\mathcal{L}^{-1}\{G(\sqrt{s})\}=\frac{1}{2 t \sqrt{\pi t}} \int_{0}^{\infty} w e^{-\frac{w^{2}}{4 t}}\left(\frac{e^{-\lambda w}}{2 w \sqrt{\pi w}} \int_{0}^{\infty} u e^{\frac{-u^{2}}{4 w}} J_{0}(k \sqrt{u}) d u\right) d w .
$$

In the above relations, $J_{\nu}(\cdot)$ stands for the Bessel's function of the first kind of order $\nu$.

Lemma 1.18. The following relation holds true.

$$
\mathcal{L}^{-1}\left\{\int_{0}^{a} \frac{e^{-\eta\left(1+\frac{w}{\sqrt{s}+w}\right)}}{\sqrt{s}+w} d \eta\right\}=\frac{1}{2 \sqrt{\pi t^{3}}} \int_{0}^{\infty} \xi e^{-\frac{\xi^{2}}{4 t}}\left(\int_{0}^{a} e^{-\eta} J_{0}(2 \sqrt{w \xi \eta}) d \eta\right) d \xi .
$$

Proof. Let us take

$$
F(s)=\int_{0}^{a} \frac{e^{-\eta\left(1+\frac{w}{s+w}\right)}}{s+w} d \eta
$$

Then, we have

$$
F(\sqrt{s})=\int_{0}^{a} \frac{e^{-\eta\left(1+\frac{w}{\sqrt{s}+w}\right)}}{\sqrt{s}+w} d \eta
$$

By using the complex inversion formula, we get

$$
\mathcal{L}^{-1}\{F(s)\}=\frac{1}{2 \pi i} \int_{c-i \infty}^{c+i \infty}\left(\int_{0}^{a} \frac{e^{-\eta\left(1+\frac{w}{s+w}\right)}}{s+w} d \eta\right) e^{s t} d s
$$


changing the order of integration which is permissible, to obtain

$$
\mathcal{L}^{-1}\{F(s)\}=\int_{0}^{a} e^{-\eta}\left(\frac{1}{2 \pi i} \int_{c-i \infty}^{c+i \infty} \frac{e^{-\eta\left(\frac{w}{s+w}\right)}}{s+w} e^{s t} d s\right) d \eta
$$

Let us introduce a change of variable $z=s+w$ in the inner integral to get

$$
\mathcal{L}^{-1}\{F(s)\}=\int_{0}^{a} e^{-\eta}\left(\frac{1}{2 \pi i} \int_{c^{\prime}-i \infty}^{c^{\prime}+i \infty} \frac{e^{-\eta\left(\frac{w}{z}\right)}}{z} e^{z t} d z\right) e^{-w t} d \eta .
$$

The value of inner integral is $J_{0}(2 \sqrt{w t \eta})$ so that

$$
\mathcal{L}^{-1}\{F(s)\}=\int_{0}^{a} e^{-\eta} J_{0}(2 \sqrt{w t \eta}) d \eta .
$$

At this point, application of Efros's theorem leads to the following result

$$
\mathcal{L}^{-1}\{F(\sqrt{s})\}=\frac{1}{2 \sqrt{\pi t^{3}}} \int_{0}^{\infty} \xi e^{-\frac{\xi^{2}}{4 t}}\left(\int_{0}^{a} e^{-\eta} J_{0}(2 \sqrt{w \xi \eta}) d \eta\right) d \xi .
$$

\section{EVAluAtion OF CERTAIN TRIGONOMETRIC INTEGRALS VIA THE LAPLACE TRANSFORM}

An interesting application of Laplace transform involves the evaluation of certain integrals, particularly those containing a free parameter. Some integrals may be evaluated by first taking the Laplace transform of the integrand with respect to a free parameter. The resulting integral is hopefully easier to evaluate than the original and, by applying the inverse Laplace transform, we obtain our desired result.

Theorem 2.1. The following integral relation holds true.

$$
\int_{0}^{\infty} \sin x t \sin \left(\frac{1}{t}\right) \frac{d t}{t^{\nu+2}}=\frac{\pi}{2} x^{\left(\frac{\nu}{2}\right)+1} J_{\nu+2}(2 \sqrt{x}), \quad \nu=0,1,2,3, \ldots
$$

Proof. Let us assume that

$$
I(x)=\int_{0}^{\infty} \sin x t \sin \left(\frac{1}{t}\right) \frac{d t}{t^{\nu+2}} .
$$

Taking the Laplace transform of the above integral, we obtain

$$
\mathcal{L}\{I(x)\}=K(s)=\int_{0}^{\infty} \mathcal{L}\{\sin t x\} \sin \left(\frac{1}{t}\right) \frac{d t}{t^{\nu+2}} .
$$

After simplifying, we arrive at

$$
K(s)=\int_{0}^{\infty} \frac{1}{t^{2}+s^{2}} \sin \left(\frac{1}{t}\right) \frac{d t}{t^{\nu+1}} .
$$

In order to evaluate the above integral, by introducing the change of variable $t^{-1}=\frac{\xi}{s}$, we obtain

$$
K(s)=\frac{1}{s^{\nu+3}} \int_{0}^{\infty} \frac{\xi^{\nu+1}}{\xi^{2}+1} \sin \left(\frac{\xi}{s}\right) d \xi
$$


At this point, we use the following well known integral identity

$$
\int_{0}^{\infty} \frac{\cos r u}{u^{2}+1} d u=\frac{\pi}{2} e^{-r}
$$

By Leibniz's rule, after $\nu+1$ times differentiation with respect to $r$, we get

$$
\int_{0}^{\infty} \frac{u^{\nu+1} \sin r u}{u^{2}+1} d u=\frac{\pi}{2} e^{-r},
$$

replacing $r$ by $\frac{1}{s}$ in the above equation, we obtain

$$
K(s)=\mathcal{L}\{I(x)\}=\frac{\pi}{2} \frac{e^{-\frac{1}{s}}}{s^{\nu+3}},
$$

hence, we get

$$
I(x)=\mathcal{L}^{-1}\left\{\frac{\pi}{2} \frac{e^{-\frac{1}{s}}}{s^{\nu+3}}\right\}=\frac{\pi}{2} x^{\frac{\nu}{2}+1} J_{\nu+2}(2 \sqrt{x}) .
$$

In the special case of $\nu=0, x=1$, we have

$$
I(1)=\frac{2}{\pi} \int_{0}^{\infty} \sin t \sin \left(\frac{1}{t}\right) \frac{d t}{t^{2}}=J_{2}(2) .
$$

\section{Main Results}

In this section, the author considered certain non-homogeneous time fractional partial differential equations which are a generalization of the Cauchy problem. In this study, only the Laplace transformation is considered as it is easily understood and being popular among engineers and scientists. The basic goal of this paper is to employ the Laplace transform method for studying the above mentioned problem. The goal has been achieved by formally deriving the exact solution. Transform method introduces a significant improvement in this field over the existing techniques.

\section{Solution to non-homogeneous partial fractional differential equation with constant coefficients}

In recent years, it has turned out that many phenomena in fluid mechanics, physics, biology, engineering and other areas of the sciences can be successfully modeled by the use of fractional derivatives. That is because of a realistic modeling of a physical phenomenon having dependence not only at the time instant, but also the previous time history can be successfully achieved by using fractional calculus. Fractional differential equations arise in the unification of diffusion and wave propagation phenomenon. The time fractional heat equation, which is a mathematical model of a wide range of important physical phenomena, is a partial differential equation obtained from the classical heat equation by replacing the first time derivative of a fractional derivative of fractional order in the Caputo sense [14]. The Laplace transforms is very useful in finding the solution to initial value problems described by linear PDEs. The following example of application illustrates the method of the transforms. 
Problem 3.1. (Non-homogeneous sub-ballistic fractional PDE with constant coefficients) Let us solve the following Cauchy type non-homogeneous partial fractional differential equation with constant coefficients. The special case of the problem was considered by V. V. Uchaikin [18].

$$
\begin{gathered}
D_{t}^{c, \alpha} u+\frac{\partial u}{\partial x}-k u=\lambda f(x), \quad 0<\alpha \leq 1, \quad x, t>0, \\
u(x, 0)=\beta .
\end{gathered}
$$

Solution. We define the Laplace transform of $u(x, t)$ by

$$
U(x, s)=\int_{0}^{\infty} u(x, t) e^{-s t} d t .
$$

Application of the Laplace transforms leads to the solution of the transformed problem in the following form

$$
\frac{\partial U}{\partial x}+\left(s^{\alpha}-k\right) U=\beta s^{\alpha-1}+\frac{\lambda f(x)}{s} .
$$

The above equation has the following solution

$$
U(x, s)=\frac{\beta s^{\alpha-1}}{s^{\alpha}-k}+\lambda \frac{e^{-\left(s^{\alpha}-k\right) x}}{s} \int_{0}^{x} e^{\left(s^{\alpha}-k\right) \eta} f(\eta) d \eta .
$$

In order to invert the above relation, let us rewrite the above equation as follows

$$
U(x, s)=\frac{\beta s^{\alpha-1}}{s^{\alpha}-k}+\lambda e^{k x} \int_{0}^{x} \frac{e^{-(x-\eta) s^{\alpha}}}{s} e^{-k \eta} f(\eta) d \eta .
$$

Taking the inverse Laplace transform of the above relation, leads to the following result

$$
u(x, t)=\mathcal{L}^{-1}\left\{\frac{\beta s^{\alpha-1}}{s^{\alpha}-k}\right\}+\lambda e^{k x} \int_{0}^{x} \mathcal{L}^{-1}\left\{\frac{e^{-(x-\eta) s^{\alpha}}}{s}\right\} e^{-k \eta} f(\eta) d \eta .
$$

In view of the Corollary 1.7 and Definition 1.3 , we arrive at

$$
\begin{aligned}
& u(x, t)=E_{\alpha, 1}\left(k t^{\alpha}\right) \\
& +\lambda e^{k x} \int_{0}^{x}\left(\int_{0}^{\infty} \frac{e^{-t r-(x-\eta) r^{\alpha} \cos \pi \alpha}}{\pi r} \sin \left((x-\eta) r^{\alpha} \sin (\pi \alpha)\right) d r\right) e^{-k \eta} f(\eta) d \eta .
\end{aligned}
$$

At this point, let us consider the the special case of $\alpha=0.5$. From relation (3.1) we obtain

$$
U(x, s)=\frac{\beta}{\sqrt{s}(\sqrt{s}-k)}+\lambda \frac{e^{-(\sqrt{s}-k) x}}{s} \int_{0}^{x} e^{(\sqrt{s}-k) \eta} f(\eta) d \eta .
$$

Finally, after taking the inverse Laplace transform, we get

$$
u(x, t)=\mathcal{L}^{-1}\left\{\frac{\beta}{\sqrt{s}(\sqrt{s}-k)}\right\}+\lambda e^{k x} \int_{0}^{x} e^{-k \eta} \mathcal{L}^{-1}\left\{\frac{e^{-(x-\eta) \sqrt{s}}}{s}\right\} f(\eta) d \eta
$$

or

$$
u(x, t)=\beta e^{k^{2} t} \operatorname{Erfc}(-k \sqrt{t})+\lambda e^{k x} \int_{0}^{x} e^{-k \eta} \operatorname{Erfc}\left(\frac{x-\eta}{2 \sqrt{t}}\right) f(\eta) d \eta .
$$


For the special case of $f(x)=\delta(x-c)$, we get the following impulsive time fractional partial differential equation

$$
\begin{gathered}
D_{t}^{c, \frac{1}{2}} u+\frac{\partial u}{\partial x}-k u=\lambda \delta(x-c), \quad 0<\alpha \leq 1, \quad x, t>0, \\
u(x, 0)=\beta
\end{gathered}
$$

with the below solution

$$
u(x, t)=\beta e^{k^{2} t} \operatorname{Erfc}(-k \sqrt{t})+\lambda e^{k x} \int_{0}^{x} e^{-k \eta} \operatorname{Erfc}\left(\frac{x-\eta}{2 \sqrt{t}}\right) \delta(\eta-c) d \eta
$$

or

$$
u(x, t)=\beta e^{k^{2} t} \operatorname{Erfc}(-k \sqrt{t})+\lambda e^{k c} \operatorname{Erfc}\left(\frac{x-c}{2 \sqrt{t}}\right) .
$$

Note. It is easy to verify that $u(x, 0)=\beta$.

\section{CONCLUSION}

The paper is devoted to studying and application of the Laplace transform to solving certain systems of time fractional partial differential equations and evaluation of integrals of Bessel's functions. The author considered a generalization of the problem of sub-ballistic fractional PDE with constant coefficients, studied by V. V. Uchaikin. The transform method provides a powerful method for analyzing linear systems. The main purpose of this paper is to develop a method for finding analytic solutions of the system of integro-differential equations and time fractional PDEs.

\section{ACKNOWLEDGMENTS}

The author would like to thank the referees for careful and thoughtful readings of the manuscript, which has helped to improve the presentation of the results.

\section{RefERENCES}

[1] A. Aghili, New results involving Airy polynomials, fractional calculus and solution to generalized heat equation, New Trends in Mathematical Sciences 3 (2015), 133-143.

[2] A. Aghili, Fractional Black-Scholes equation, J. Finan. Eng. 4 (2017), 15 pp.

[3] A. Apelblat, Laplace transforms and their applications, Nova Science Publishers Inc., New York, 2012.

[4] T. M. Atanacković and B. Stanković, Dynamics of a viscoelastic rod of fractional derivative type, Z. Angew. Math. Mech. 82 (2002), 377-386.

[5] T. M. Atanacković and B. Stanković, On a system of differential equations with fractional derivatives arising in rod theory, J. Phys. A, Math. Gen. 37 (2004), 1241-1250.

[6] R.S. Dahiya and M. Vinayagamoorthy, Laplace transfom pairs of $n$-dimensions and heat conduction problem, Math. Comput. Modelling 13 (1990), 35-50.

[7] V. A. Ditkin and A. P. Prudnikov, Operational calculus in two variables and its application, Pergamon Press, New York, 1962.

[8] A. A.Kilbas, H. M. Srivastava and J. J.Trujillo, Theory and Applications of Fractional Differential Equations, North Holland Mathematics Studies 204, Elsevier, Amesterdam, 2006.

[9] Yu. F. Luchko and H. M. Srivastava, The exact solution of certain differential equations of fractional order by using operational calculus, Comput. Math. Appl. 29 (1995), 73-85. 
[10] K. S. Miller and B. Ross, An Introduction to the Fractional Calculus and Fractional Differential Equations, John Wiley \& Sons, New York, 1993.

[11] K. B. Oldham and J. Spanier, The Fractional Calculus, Theory and Applications of Differentiation and Integration to Arbitrary Order, Mathematics in Science and Engineering 111, Academic Press, New York, London, 1974.

[12] K. B. Oldham and J. Spanier, Fractional calculus and its applications, Bull. Inst. Politeh. Iaşi, Secţ. I 24 (1978), 29-34.

[13] I. Podlubný, The Laplace transform method for linear differential equations of fractional order, Slovak Academy of Sciences, Košice, 1994.

[14] I. Podlubný, Fractional differential equations, Academic Press, San Diego, CA, 1999.

[15] G. Samko , A. Kilbas and O. Marchiev, Fractional Integrals and derivatives theory and Applications, Gordon and Breach, Amesterdam, 1993.

[16] W. R. Schneider and W. Wyss, Fractional diffusion and wave equations, J. Math. Phys. 30 (1989), 134-144.

[17] B. A. Stanković, System of partial differential equations with fractional derivatives, Mat. Vesn. 54 (2002), 187-194.

[18] V. V. Uchaikin, Fractional Derivatives for Physicists and Engineers, Volume I: Background and Theory, Nonlinear Physical Science, Springer, Berlin, 2012.

[19] W. Wyss, The fractional diffusion equation, J. Math. Phys. 27 (1986), 2782-2785.

Arman Aghili, University of Guilan, Faculty of Mathematical sciences, Department of Applied Mathematics, Rasht - Iran, P.O. Box 1841

e-mail: ar.aghili@hotmail.com, arman.aghili@gmail.com 
